\title{
Abstract: An SVR-Based Data-Driven Leaflet Modeling Approach for Personalized Aortic Valve Prosthesis Development
}

\author{
Jannis Hagenah ${ }^{1}$, Tizian Evers ${ }^{1}$, Michael Scharfschwerdt ${ }^{2}$, Achim Schweikard ${ }^{1}$, \\ Floris Ernst ${ }^{1}$ \\ ${ }^{1}$ Institute for Robotics and Cognitive Systems, University of Lübeck \\ ${ }^{2}$ Department of Cardiac Surgery, University Hospital Schleswig-Holstein, Lübeck \\ hagenah@rob.uni-luebeck.de
}

While the aortic valve geometry is highly patient-specific and studies indicate its high influence on the circulation, state-of-the-art valve prostheses are not aiming at reproducing this individual geometry. One challenge in manufacturing personalized prostheses is the imaging of the thin leaflets in their curved 3D shape as well as the mapping from this shape to the planar $2 \mathrm{D}$ leaflet shape that is cut out of the fabrication material. Even in the gold standard imaging modality (transesophageal ultrasound), the leaflets are barely visible. Hence, we present a machine learning approach to estimate the individual leaflet shape from the image information on the shape of the surrounding tissue, i.e. the aortic root.

Thus, a database was set up to derive and evaluate valve leaflet shape models [1] . First, 3D ultrasound images of ex-vivo porcine valves were acquired under physiologically realistic pressure. In these images, geometric key features were identified manually to describe the individual geometry of the root. In a second step, the valves' leaflets were cut out, spread on an illuminated plate and photographed in this state. From these images, the leaflet shape was extracted using edge detection.

This database allows the derivation of a data-driven leaflet model utilizing non-linear support vector regression (SVR), aiming on a mapping from the geometric key features to the leaflet shape. Additionally, an existing, hand-crafted geometric leaflet shape model was evaluated on the dataset to evaluate its performance regarding personalization. The data-driven approach provided an acceptable leaflet shape estimation ( $0.61 \mathrm{~mm}$ ASCD) and clearly outperformed the existing model (2.21 mm ASCD). Hence, machine learning is capable of estimating the individual leaflet shape from sparse image data. This presents an important step towards personalized aortic valve prostheses.

\section{References}

1. Hagenah J, Evers T, Scharfschwerdt M, et al. An SVR-based data-driven leaflet modeling approach for personalized aortic valve prosthesis development. Comput Cardiol. 2018; p. accepted. 